\title{
Transgastric natural orifice transluminal endoscopic surgery (NOTES) to remove a foreign body from the abdominal cavity of a 12-year old boy
}

A 12-year-old boy was admitted to our hospital with sharp upper abdominal pain for the previous 5 days. Physical examination revealed epigastric tenderness but no rebound pain. The boy revealed that, 1 year previously, he had swallowed some sharp metal and magnetic objects, without ever telling either his parents or anyone else.

After his admission, a computed tomography (CT) scan was performed, which showed multiple masses and long strips of high density shadow on the greater curvature of the stomach, some of which appeared to be outside the gastric cavity ( $\vee$ Fig. 1). Gastroscopy showed two clumpy foreign bodies (approximately $6 \times 4 \mathrm{~cm}$ in size) in the gastric cavity, with many long foreign body strips attached, and that the stomach wall was swollen and eroded ( $\mathbf{F i g .} \mathbf{2}$ a). A total of 19 iron nails and eight magnets shaped like rugby balls were removed using a pair of foreign body forceps during the endoscopy ( Fig. $\mathbf{2 b}$ b). However, when a repeat CT scan was performed on the second day after surgery, it was found that there was still an iron nail of approximately $5 \mathrm{~cm}$ in length in the child's abdominal cavity ( Fig.3). This iron nail had passed through the gastric wall and fallen into the abdominal cavity, where it was assumed to have remained between the posterior wall of the stomach and the pancreas for approximately 1 year.

After multidisciplinary consultations with pediatric medicine, pediatric surgery, gastrointestinal surgery, gastroenterology, and other disciplines, we decided to remove the foreign body using natural orifice transluminal endoscopic surgery (NOTES). NOTES is an evolving minimally invasive surgery that aims to eliminate abdominal trauma and its related complications [1]. Compared with traditional surgery and laparoscopy, it has many advantages, including no scarring, less trauma, less pain, and a shorter surgical path $[2,3]$.
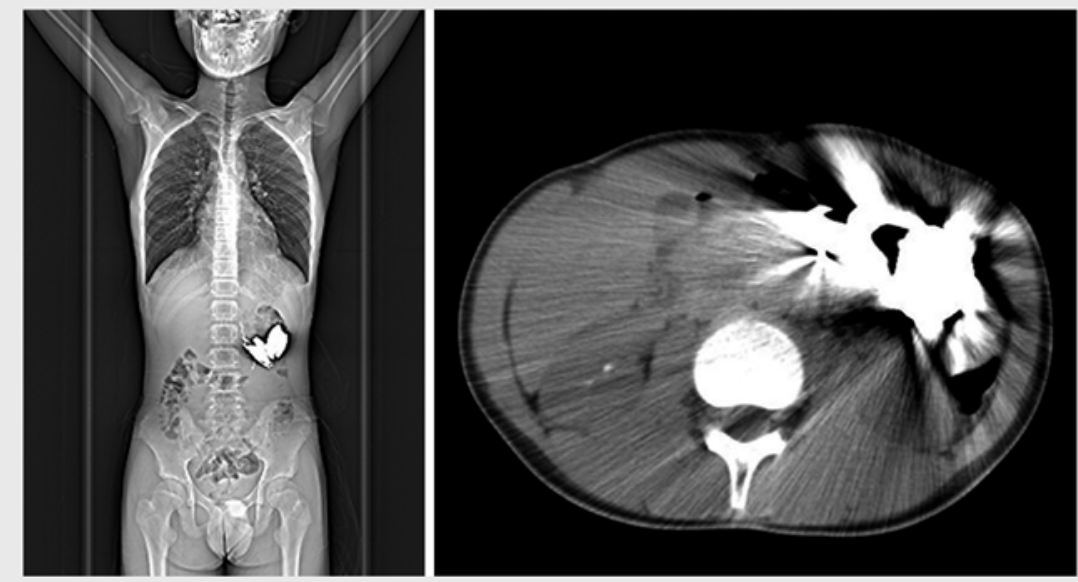

Fig. 1 Computed tomography (CT) scan in a 12-year-old boy with upper abdominal pain showing multiple masses and long strips of high density shadow on the greater curvature of the stomach.
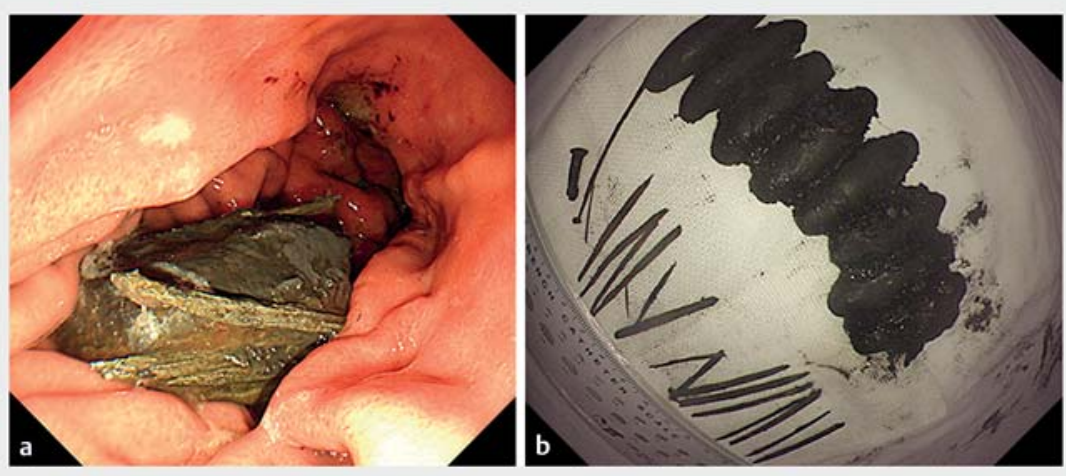

- Fig. 2 Appearance of one of the foreign bodies: a on endoscopy, with a large clump of foreign material seen in the gastric cavity; $\mathbf{b}$ following removal, when it was seen to consist of magnets and iron nails that had been swallowed by the boy 1 year previously.

The patient's parents signed an informed consent form for NOTES and the surgery was performed with the boy under endotracheal intubation and anesthesia. The procedure was performed in the following steps (-Fig.4; Video 1). (i) The locations of the foreign body and the gastric wall were detected using endoscopic ultrasonography (EUS) and an in- cision was made in the greater curvature of the middle part of the gastric body near the posterior wall. The entire layer of the gastric wall was incised using a DualKnife and IT-knife. (ii) The endoscope was passed into the small abdominal cavity, where extensive adhesions and wrappings were found between the posterior gastric wall and the pancreas; 
however, no foreign body could be seen. (iii) The adhesions were separated and incised with the DualKnife and IT-knife. (iv) The foreign body was located using EUS, which showed that one end of it had become partially inserted into the pancreas. It was completely separated and loosened, and was then removed using foreign body forceps through the incision in the gastric wall. It was found to be a $4.8-\mathrm{cm}$ iron nail. (v) The wound was treated with hot coagulation for-
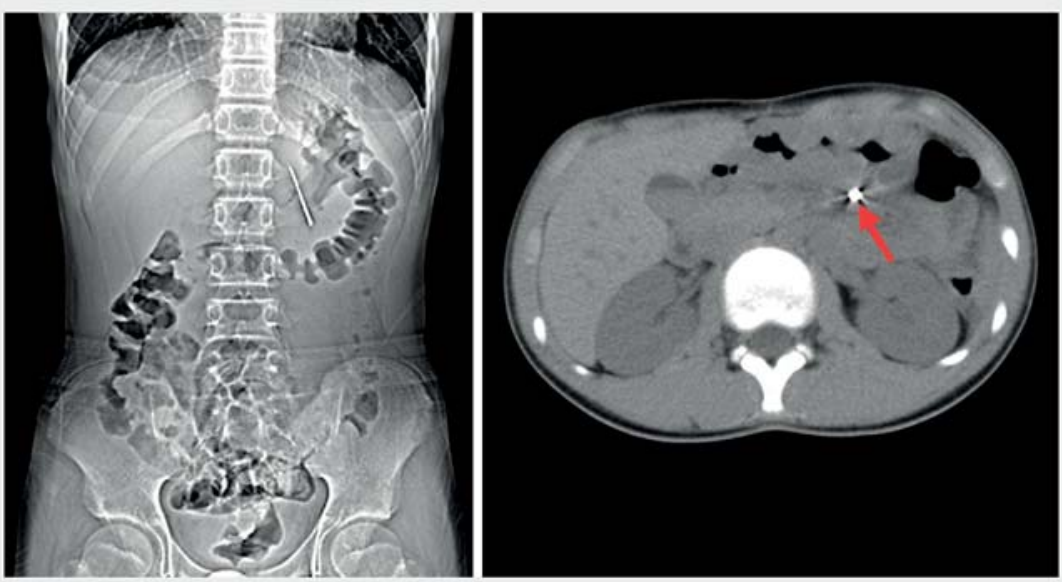

ceps, and the abdominal cavity was washed with metronidazole; the incision in the gastric wall was then sutured with endoclips and nylon loops.

The surgery was completely successful, and the patient had no abdominal pain, fever, or other discomfort after the operation. He recovered and was discharged on the fifth day after surgery.

Endoscopy_UCTN_Code_TTT_1AO_2AN

Competing interests

The authors declare that they have no conflict of interest.

- Fig. 3 Repeat computed tomography (CT) scan showing a long strip of high density shadow still visible at the lower segment of the greater curvature of the stomach, but on the outside of the gastric cavity (arrow).
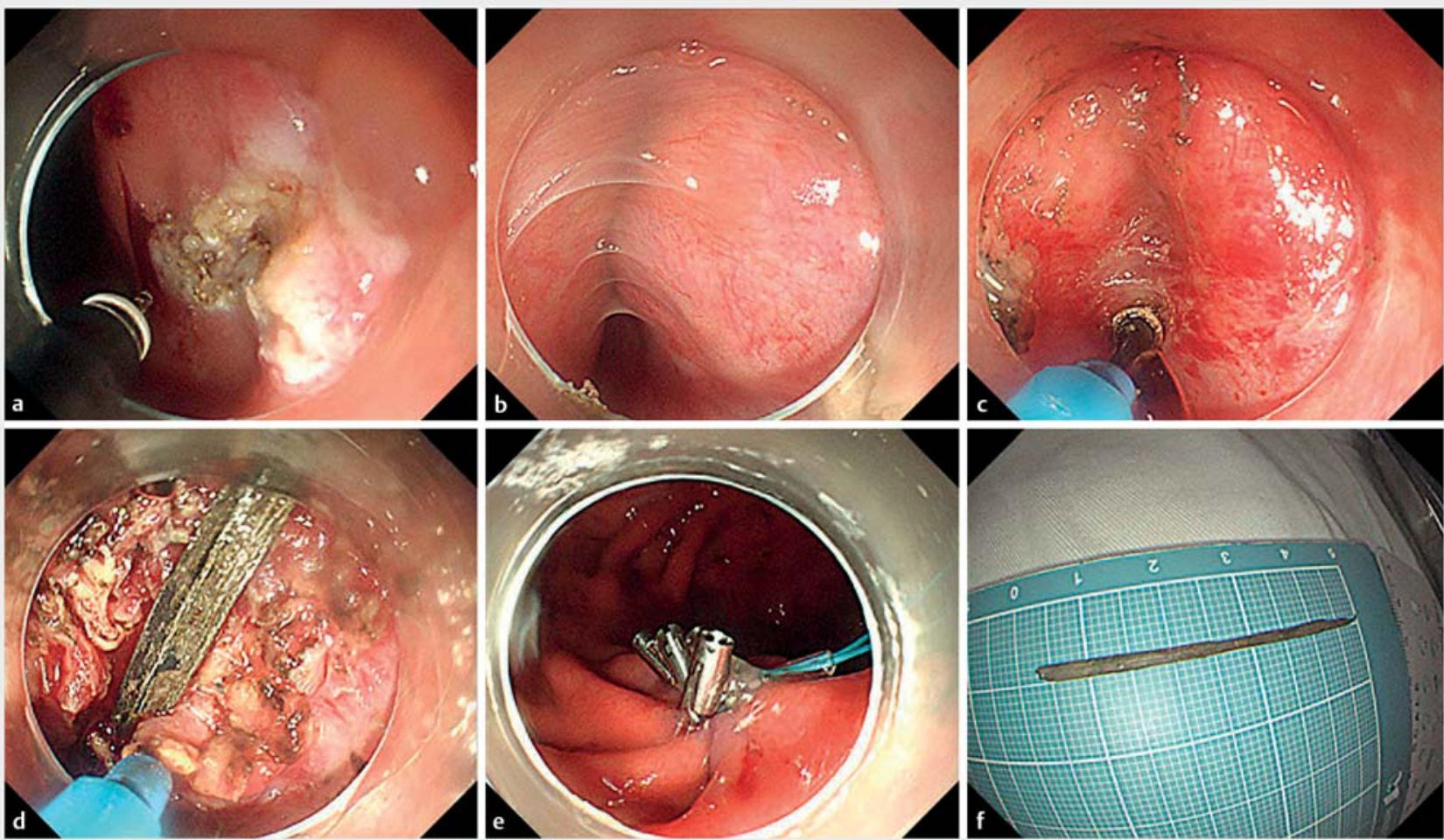

- Fig. 4 Steps in the transgastric natural orifice transluminal endoscopic surgery (NOTES) performed to remove the foreign body from the abdominal cavity included: a incision of the gastric wall; $\mathbf{b}$ passage of the endoscope into the abdominal cavity; $\mathbf{c}$ separation and incision of the celiac adhesions; $\mathbf{d}$ finding and loosening the foreign body; e gastric closure using endoclips and nylon loops. $\mathbf{f}$ The foreign body was an iron nail. 


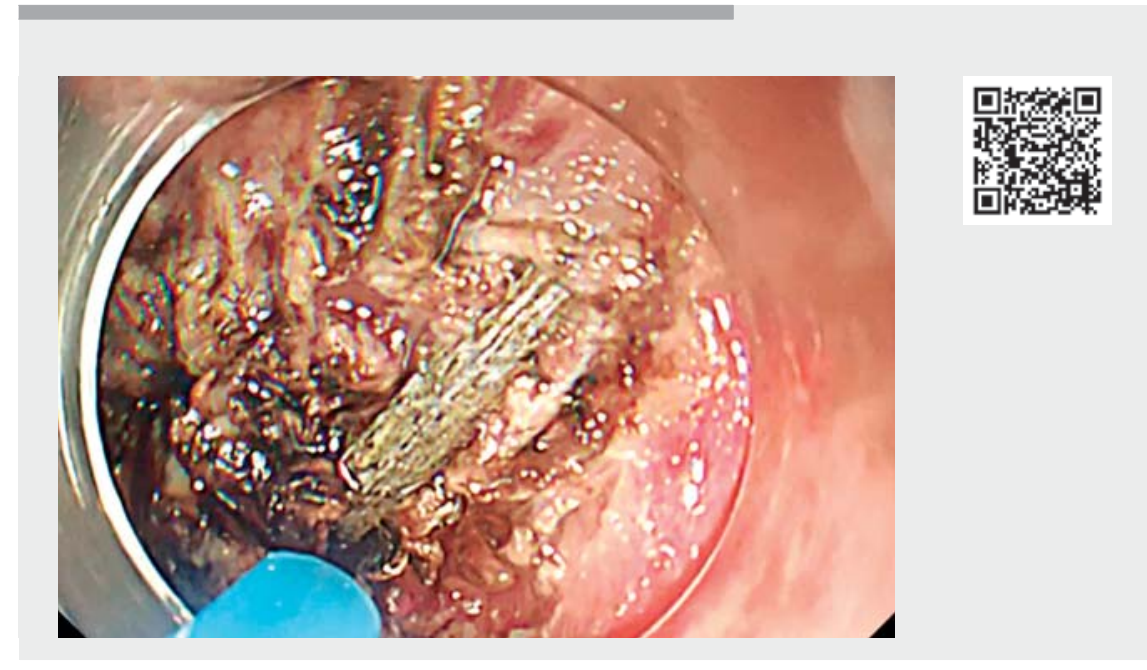

$\checkmark$ Video 1 Transgastric natural orifice transluminal endoscopic surgery (NOTES) to remove a foreign body from the abdominal cavity.

The authors

\section{Shengbing Wang*, Fu Guan*, Fuqun Wang, Pingwu Wen \\ Department of Gastroenterology, Meizhou People's Hospital, Meizhou, Guangdong Province, China}

\section{Corresponding author}

\section{Shengbing Wang, MD}

Department of Gastroenterology, Meizhou People's Hospital, No. 63, Huangtang Road, Meizhou 514031, Guangdong Province,

China

wangshengbingyx@163.com

\section{Bibliography}

Endoscopy 2022; 54: E110-E112

DOI 10.1055/a-1407-9775

ISSN 0013-726X

published online 30.3 .2021

(c) 2021. Thieme. All rights reserved.

Georg Thieme Verlag KG, Rüdigerstraße 14,

70469 Stuttgart, Germany

\section{ENDOSCOPY E-VIDEOS \\ https://eref.thieme.de/e-videos}

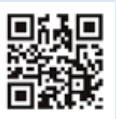

Endoscopy E-Videos is an open access online section, reporting on interesting cases and new techniques in gastroenterological endoscopy. All papers include a high quality video and all contributions are freely accessible online. Processing charges apply (currently EUR 375), discounts and wavers acc. to HINARI are available.

This section has its own submission website at

https://mc.manuscriptcentral.com/e-videos
[2] Marescaux J, Dallemagne B, Perretta S et al. Surgery without scars: report of transluminal cholecystectomy in a human being. Arch Surg 2007; 142: 823-826

[3] Bowman DE. ASGE/SAGES Working Group on Natural Orifice Translumenal Endoscopic Surgery: White Paper October 2005. Gastrointest Endosc 2006; 63: 199-203

1] Liu BR, Cui GX, Zhang XY et al. Pure transgastric NOTES ovarian cystectomy: the first human procedure. Endoscopy 2014; 46 : E199-E200 